\title{
LEAKAGE RATES FOR CRYOLAB VALVES
}

DØ Engineering Note: 3740.510-EN-192

J. M. Wendlandt

November 2, 1988

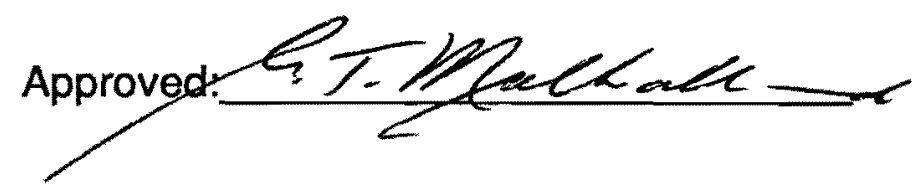


This note summarizes a brief study on the leakage rates of cryolab valves due to the failure of the valve packings. The $\Delta r$ gap between the shaft and the packing, the fluid( $\operatorname{Ar}$ or N2), the $\Delta p$ (atm) between the fluid and atmosphere, and the initial temperature of the escaping gas were varied to determine the resulting leakage rate. The heat leak in watts required to vaporize the liquid which provides the indicated flow rates is also included in the results. The following equation with the units indicated on the spread sheets was used for the calculations:

\section{$\dot{M}=\sqrt[1.8]{\frac{\Delta P}{\Delta L}\left(\frac{1}{4.98 \times 10^{-9}}\right) \frac{P}{(\mu)^{0.2}}\left(r_{1}^{2}-r_{2}^{2}\right)^{1.8}\left(r_{1}-r_{2}\right)^{1.2}}$ Units: atm, cm, g, s}

The dimensions used in the calculations were acquired over the telephone and/or found on the enclosed drawing from Cryolab. There is no implication that Cryolab was any better or worse than other valve manufacturers. They were kind enough to provide the detail needed for this study. The fluid properties were found in Ar and N2 Property handbooks. The results of this study are shown graphically and listed on accompanying spread sheets. The spread sheets and graphs can be found on the disc JW4 in the MassFlow folder. 


\begin{tabular}{|c|c|c|c|c|c|c|c|c|c|}
\hline Temperature & $\Delta P(a t m)$ & $\Delta \mathrm{L}(\mathrm{cm})$ & dens. $(\mathrm{g} / \mathrm{cm} 3)$ & $\mathrm{g} / \mathrm{cm}-\mathrm{s}$ & $\mathrm{r} 1 \quad(\mathrm{~cm})$ & $\mathrm{r} 2(\mathrm{~cm})$ & $M(g / s)$ & $\mathrm{ft} 3 / \mathrm{min}$ & Watts to \\
\hline $300 \mathrm{~K} \mathrm{~N} 2$ & 1 & 5.715 & 0.0017077 & 0.00017962 & 0.6223 & 0.62103 & 79256 & 0.04055595 & 4.36 \\
\hline $300 \mathrm{~K} \mathrm{Ar}$ & 1 & 5.715 & 0.002413 & 0.0002291 & 0.6223 & 0.62103 & 0.02570286 & 0.03382496 & 5.14 \\
\hline $90 \mathrm{~K} \mathrm{Ar}$ & 1 & 5.715 & 0.008273 & 0.0000758 & 0.6223 & 0.62103 & 0.05762822 & 0.07583874 & 11.53 \\
\hline $80 \mathrm{~K} \mathrm{N2}$ & 1 & 5.715 & 0.00670851 & 0.00005559 & 0.6223 & 0.62103 & 0.05309122 & 0.09880277 & 10.62 \\
\hline $300 \mathrm{~K} \mathrm{~N} 2$ & 2 & 5.715 & 0.00227705 & 0.0001797 & 0.6223 & 0.62103 & 0.03757928 & 0.06993503 & 7.52 \\
\hline $300 \mathrm{~K} \mathrm{Ar}$ & 2 & 5.715 & 0.003216 & 0.0002292 & 0.6223 & 0.62103 & 0.0443108 & 0.05831301 & 8.86 \\
\hline $90 \mathrm{~K} \mathrm{Ar}$ & 2 & 5.715 & 0.010774 & 0.0000784 & 0.6223 & 0.62103 & 0.09771846 & 0.1285975 & 19.54 \\
\hline $80 \mathrm{~K} \mathrm{~N} 2$ & 2 & 5.715 & 0.00876454 & 0.00005777 & 0.6223 & 0.62103 & 0.09013784 & 0.16774651 & 18.03 \\
\hline $300 \mathrm{~K} \mathrm{~N} 2$ & 3 & 5.715 & 0.0 & 0.00017977 & 0.6223 & 0.62103 & 0.0 & 0.09 & 10.42 \\
\hline $300 \mathrm{~K} \mathrm{Ar}$ & 3 & 5.715 & 0.003855 & 0.0002293 & 0.6223 & 0.62103 & 0.0613824 & 0.08077924 & 12.28 \\
\hline $90 \mathrm{~K} \mathrm{Ar}$ & 3 & 5.715 & 0.0122747 & 0.0000803 & 0.6223 & 0.62103 & 0.13125411 & 0.1727304 & 26.25 \\
\hline $80 \mathrm{~K} \mathrm{N2}$ & 3 & 5.715 & 0.01039241 & 0.00005928 & 0.6223 & 0.62103 & 0.12376404 & 0.23032488 & 24.75 \\
\hline
\end{tabular}

$\Delta \mathrm{P}=$ "inches" 0.0005 


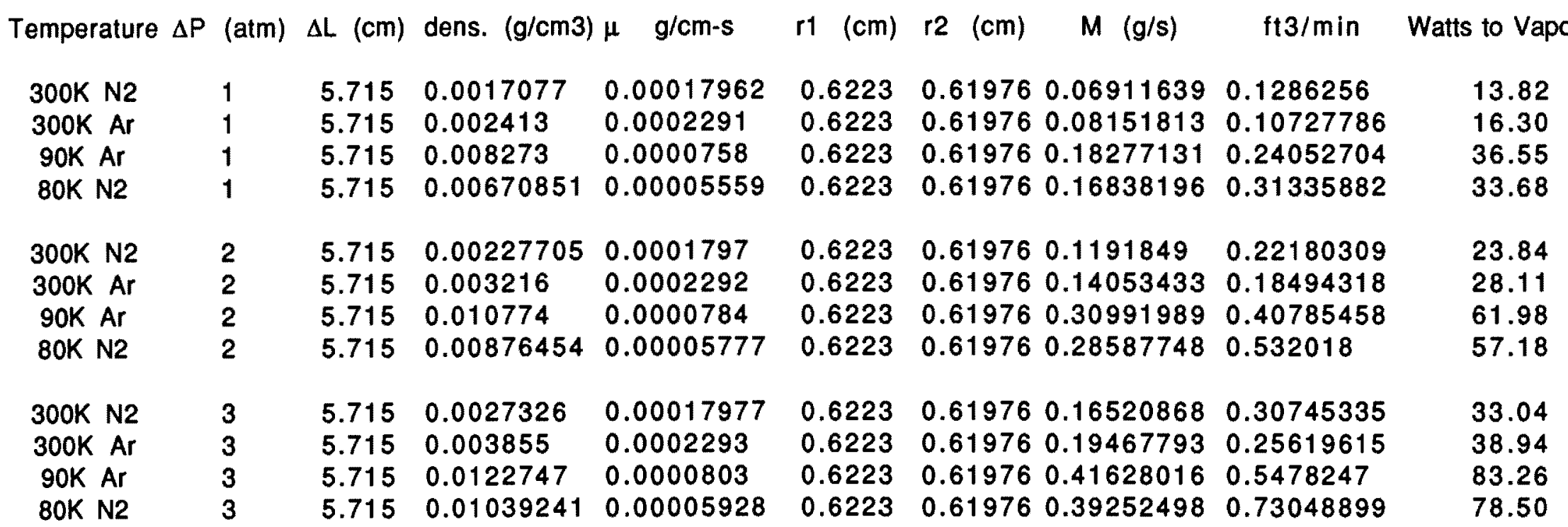

$\Delta r=$ "inches" 0.001 
Temperature $\Delta \mathrm{P}$ (atm) $\Delta \mathrm{L}(\mathrm{cm})$ dens. $(\mathrm{g} / \mathrm{cm} 3) \mu \quad \mathrm{g} / \mathrm{cm}-\mathrm{s}$

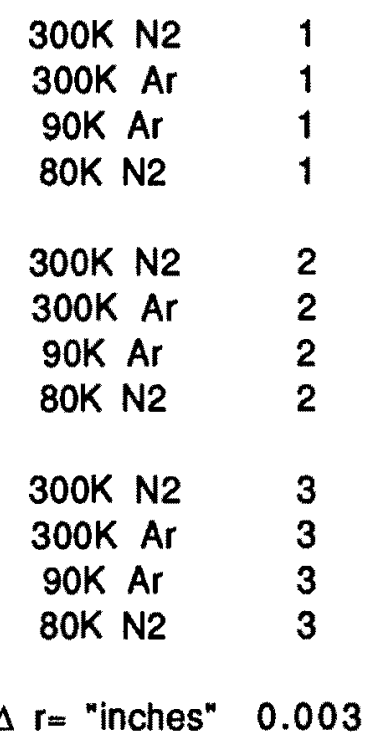

$\Delta r=$ "inches" 0.003 r1 $(\mathrm{cm})$

$12(\mathrm{~cm})$

$M(g / s)$

$\mathrm{ft} 3 / \mathrm{min}$

Watts to Vaporize
0.6223

5.715

5.715

0.00005028
0.6223

0.6223

0.6223

0.6223

0.6223

0.6223

0.6223

0.6223

0.6223

0.6223

$\begin{array}{llll}0.61468 & 0.42953964 & 0.79937327\end{array}$

$\begin{array}{llll}0.61468 & 0.50661308 & 0.66670281\end{array}$

$\begin{array}{llll}0.61468 & 1.13587415 & 1.49481039\end{array}$

$\begin{array}{lll}0.61468 & 1.04644823 & 1.94744015\end{array}$

$\begin{array}{llll}0.61468 & 0.74070183 & 1.37844611\end{array}$

$\begin{array}{llll}0.61468 & 0.87338278 & 1.14937173\end{array}$

$\begin{array}{llll}0.61468 & 1.92606813 & 2.53470566\end{array}$

$\begin{array}{llll}0.61468 & 1.77665108 & 3.30634765\end{array}$

$\begin{array}{llll}0.61468 & 1.02672715 & 1.910 ́ 73922\end{array}$

$0.61468 \quad 1.20987056 \quad 1.59218965$

$0.614682 .5870684 \quad 3.40458201$

$\begin{array}{lllll}0.6223 & 0.61468 & 2.43943639 & 4.53979112\end{array}$
85.91

101.32

227.17

209.29

148.14

174.68

385.21

355.33

205.35

241.97

517.41

487.89 
$11-8-88$

Temperature $\Delta P$ (atm) $\Delta L(\mathrm{~cm})$ dens. $(\mathrm{g} / \mathrm{cm} 3) \mu \quad \mathrm{g} / \mathrm{cm}-\mathrm{s} \quad r 1 \quad(\mathrm{~cm}) \quad r \quad(\mathrm{~cm}) \quad M \quad(\mathrm{~g} / \mathrm{s}) \quad \mathrm{ft} 3 / \mathrm{min} \quad$ Watts to Vaporize

\begin{tabular}{|c|c|c|c|c|c|c|c|c|c|}
\hline $300 \mathrm{~K} \mathrm{~N} 2$ & 1 & 5.715 & 0.0017077 & 0.00017962 & 0.6223 & 0.6096 & 1.00222202 & 1.86513519 & 200.44 \\
\hline $300 \mathrm{~K} \mathrm{Ar}$ & 1 & 5.715 & 0.002413 & 0.0002291 & 0.6223 & 0.6096 & 1.18205338 & 1.55558225 & 236.41 \\
\hline $90 \mathrm{~K} \mathrm{Ar}$ & 1 & 5.715 & 0.008273 & 0.0000758 & 0.6223 & 0.6096 & 2.65027482 & 3.48776167 & 530.05 \\
\hline $80 \mathrm{~K} \mathrm{~N} 2$ & 1 & 5.715 & 0.00670851 & 0.00005559 & 0.6223 & 0.6096 & 2.44162206 & 4.54385866 & 488.32 \\
\hline $300 \mathrm{~K} \mathrm{~N} 2$ & 2 & 5.715 & 0.00227705 & 0.0001797 & 0.6223 & 0.6096 & 1.72824024 & 3.21625509 & 345 \\
\hline $300 \mathrm{~K} \mathrm{Ar}$ & 2 & 5.715 & 0.003216 & 0.0002292 & 0.6223 & 0.6096 & 2.03781763 & 2.68176801 & 407.5 \\
\hline $90 \mathrm{~K} \mathrm{Ar}$ & 2 & 5.715 & 0.010774 & 0.0000784 & 0.6223 & 0.6096 & 4.49399246 & 5.91409407 & 898.80 \\
\hline $80 \mathrm{~K} \mathrm{~N} 2$ & 2 & 5.715 & 0.00876454 & 0.00005777 & 0.6223 & 0.6096 & 4.14536557 & 7.71452533 & 829.07 \\
\hline $300 \mathrm{~K} \mathrm{~N} 2$ & 3 & 5.715 & 0.0027326 & 0.00017977 & 0.6223 & 0.6096 & 2.39560792 & 4.45822633 & 479.12 \\
\hline $300 \mathrm{~K} \mathrm{Ar}$ & 3 & 5.715 & 0.003855 & 0.0002293 & 0.6223 & 0.6096 & 2.8229267 & 3.71497154 & 564.59 \\
\hline $90 \mathrm{~K} \mathrm{Ar}$ & 3 & 5.715 & 0.0122747 & 0.0000803 & 0.6223 & 0.6096 & 6.03626927 & 7.94373035 & 1207.2 \\
\hline $80 \mathrm{~K} \mathrm{N2}$ & 3 & 5.715 & 0.01039241 & 0.00005928 & 0.6223 & 0.6096 & 5.69180734 & 10.5924535 & 1138.36 \\
\hline
\end{tabular}

$\Delta r=$ "inches" 0.005 
Temperature $\Delta P(a t m) \Delta L(\mathrm{~cm})$ dens. $(\mathrm{g} / \mathrm{cm} 3) \mu \quad \mathrm{g} / \mathrm{cm}-\mathrm{s}$

$300 \mathrm{~K} \mathrm{~N} 2$
$300 \mathrm{~K} \mathrm{Ar}$
$90 \mathrm{~K} \mathrm{Ar}$
$80 \mathrm{~K} \mathrm{N2}$
$300 \mathrm{~K} \mathrm{N2}$
$300 \mathrm{~K} \mathrm{Ar}$
$90 \mathrm{~K} \mathrm{Ar}$
$80 \mathrm{~K} \mathrm{N2}$
$300 \mathrm{~K} \mathrm{N2}$
$300 \mathrm{~K} \mathrm{Ar}$
$90 \mathrm{~K} \mathrm{Ar}$
$80 \mathrm{~K} \mathrm{N2}$

$\Delta r=$ "inches" $\quad 0.01$ $\mathrm{r1}(\mathrm{cm}) \mathrm{r} 2(\mathrm{~cm}) \quad M(\mathrm{~g} / \mathrm{s}) \quad \mathrm{ft} 3 / \mathrm{min}$ Watts to Vaporize

$\begin{array}{lllllll}5.715 & 0.0017077 & 0.00017962 & 0.6223 & 0.5969 & 3.14905395 & 5.86038939 \\ 5.715 & 0.002413 & 0.0002291 & 0.6223 & 0.5969 & 3.71409704 & 4.88775171 \\ 5.715 & 0.008273 & 0.0000758 & 0.6223 & 0.5969 & 8.3273548 & 10.9587989 \\ 5.715 & 0.00670851 & 0.00005559 & 0.6223 & 0.5969 & 7.67175277 & 14.2771319 \\ & & & & & & \\ 5.715 & 0.00227705 & 0.0001797 & 0.6223 & 0.5969 & 5.43025559 & 10.1057056 \\ 5.715 & 0.003216 & 0.0002292 & 0.6223 & 0.5969 & 6.40297011 & 8.42630866 \\ 5.715 & 0.010774 & 0.0000784 & 0.6223 & 0.5969 & 14.1204487 & 18.5825105 \\ 5.715 & 0.00876454 & 0.00005777 & 0.6223 & 0.5969 & 13.0250379 & 24.2395955 \\ & & & & & & \\ 5.715 & 0.0027326 & 0.00017977 & 0.6223 & 0.5969 & 7.527173 & 14.008069 \\ 5.715 & 0.003855 & 0.0002293 & 0.6223 & 0.5969 & 8.86983946 & 11.6727087 \\ 5.715 & 0.0122747 & 0.0000803 & 0.6223 & 0.5969 & 18.9663938 & 24.9597742 \\ 5.715 & 0.01039241 & 0.00005928 & 0.6223 & 0.5969 & 17.8840695 & 33.2822534\end{array}$

629.81

742.82

1665.47

1534.35

1086.05

1280.59

2824.09

2605.01

1.505 .43

1773.97

3793.28

3576.81 

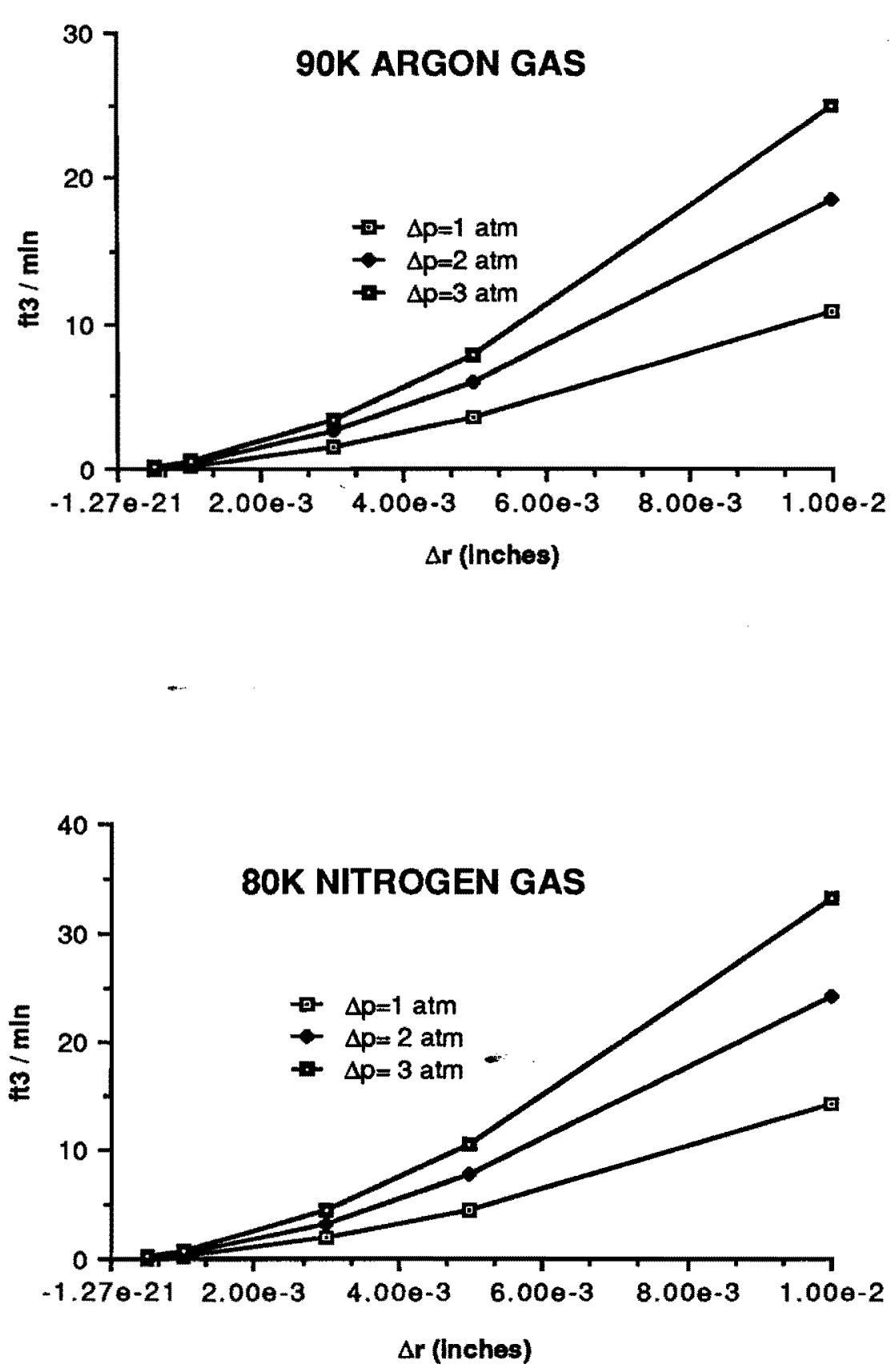

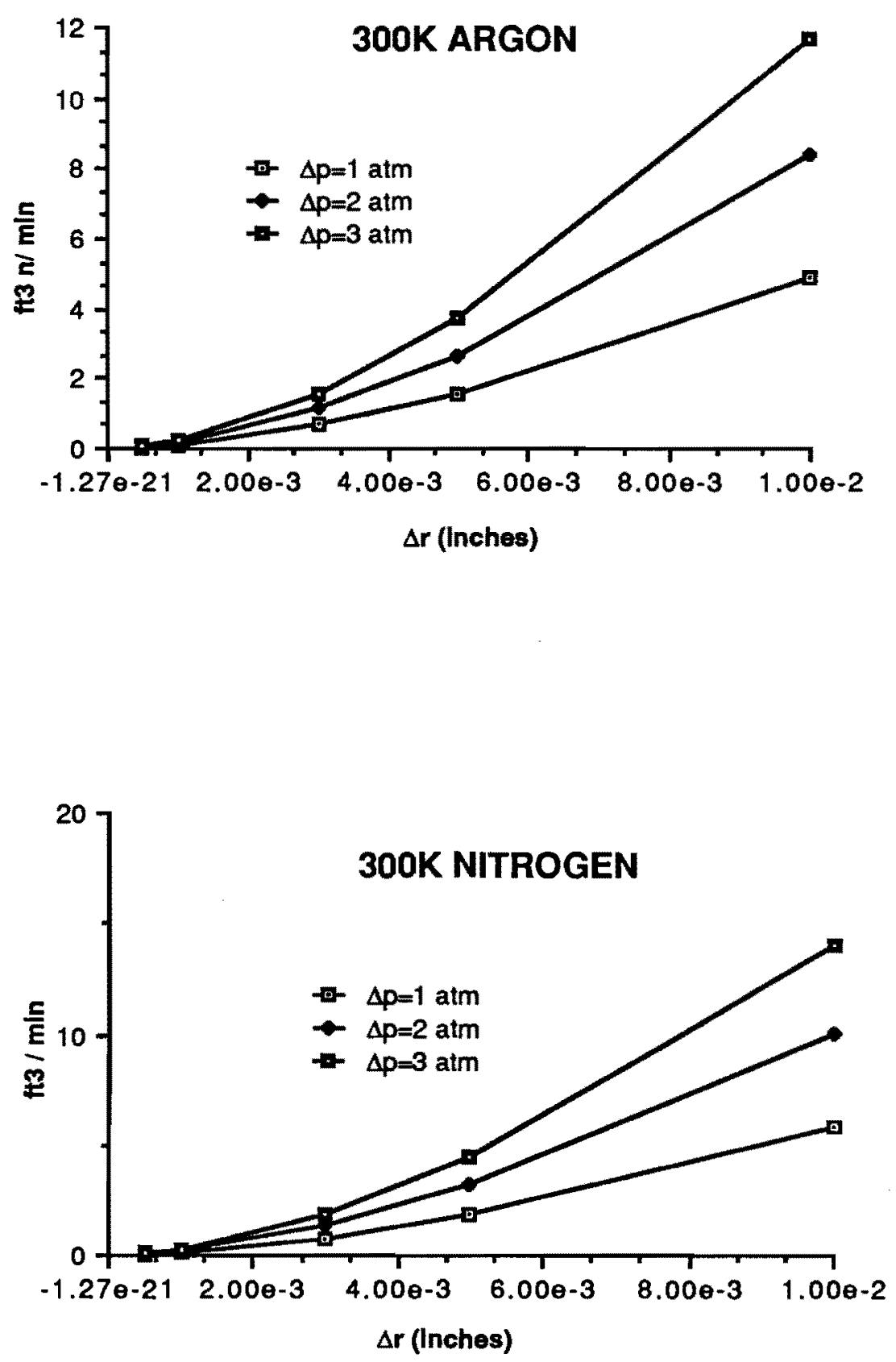

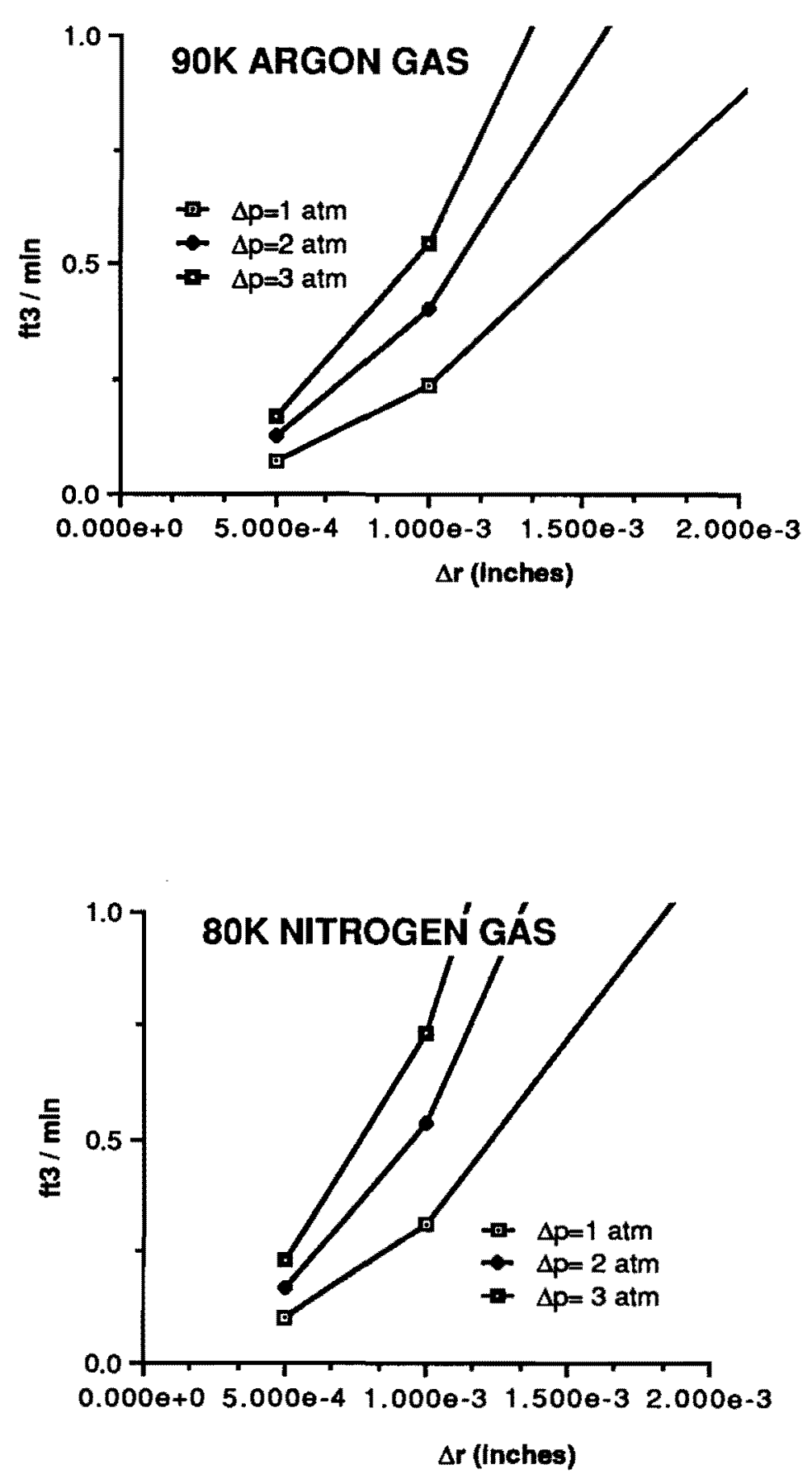

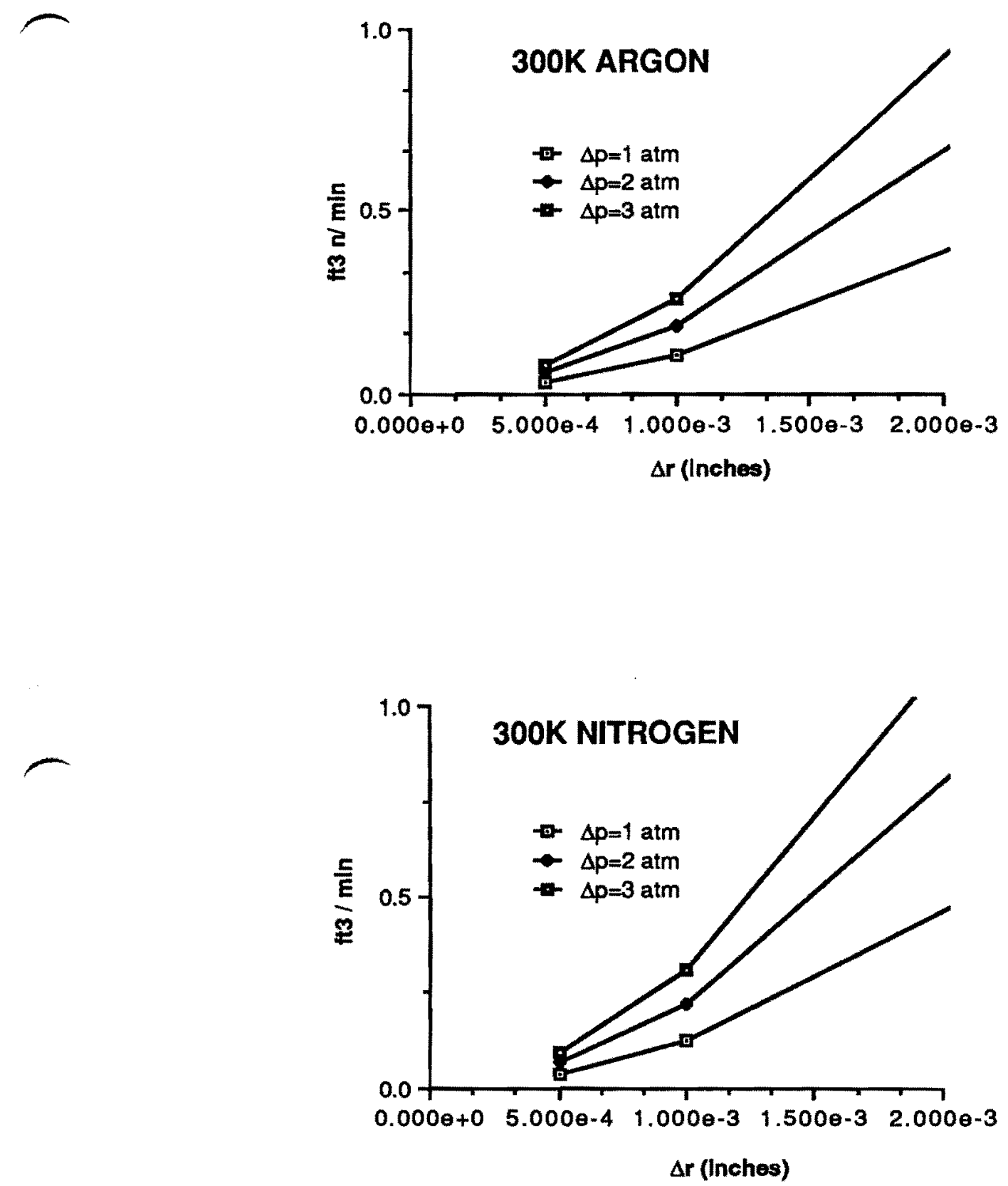